Pacific

Journal of

Mathematics

\title{
FOCAL SETS IN TWO-DIMENSIONAL SPACE FORMS
}

Carlos Arturo Escudero, Agustí Reventós and Gil Solanes 


\title{
FOCAL SETS IN TWO-DIMENSIONAL SPACE FORMS
}

\author{
Carlos Arturo Escudero, Agustí Reventós and Gil Solanes
}

We relate the area of a convex set in a 2-dimensional space of constant curvature with some integrals over the curvature radius at its boundary.

\section{Introduction}

Let $M=\partial K$ be the boundary of a compact convex domain $K$ in $\mathbb{R}^{2}$ of area $F$. Then we have the inequality

$$
\int_{M} \frac{1}{k(s)} d s \geqslant 2 F
$$

where $d s$ is the arclength measure on $M$ and $k=k(s)>0$ is the curvature of $M$ at the point of parameter $s$. Equality holds if and only if $M$ is a circle. See for instance [Escudero and Rodríguez 1996] or [Zhou 2007].

Formula (1) is the 2-dimensional analogue of Heintze and Karcher's inequality:

$$
\int_{S} \frac{1}{H} d A \geqslant 3 V
$$

where $H$ is the mean curvature of a compact embedded surface $S$ in $\mathbb{R}^{3}$ bounding a domain of volume $V$. The inequality assumes $H>0$, and equality holds if and only if $S$ is a standard sphere; see [Ros 1988; Osserman 1990].

Escudero and Reventós [2007] improved equality (1), showing

$$
\int_{M} \frac{1}{k(s)} d s=2\left(F-F_{e}\right)
$$

where $F_{e} \leq 0$ is the (algebraic) area of the domain bounded by the evolute of $M$.

Equivalently,

$$
\int_{M} \frac{\rho(s)}{2} d s=F-F_{e}
$$

where $\rho(s)=1 / k(s)$ is the curvature radius of $M$ at the point of parameter $s$.

MSC2000: primary 53C65, 53A04; secondary 52A10, 52A15, 52A55.

Keywords: curvature, focal sets, space forms, convex.

Work partially supported by DGICYT grant \#BFM2003-03458 and Universidad Tecnológica de Pereira, project 3-05-2. 
In this paper we generalize this equality to $X_{c}^{2}$, the 2-dimensional complete and simply connected riemannian manifold of constant curvature $c$, that is, for $c>0$, the sphere $\mathbb{S}_{c}^{2}$ of radius $R=1 / \sqrt{c}$ for $c>0$ or, for $c<0$, the hyperbolic plane $\mathbb{H}_{c}^{2}$ (the sphere of imaginary radius $R=-i / \sqrt{c}$ ). We assume $X_{c}^{2}$ is oriented.

Using the same techniques as in [Gallego et al. 2005], we obtain a result that coincides, for $c=0$, with formula (2). First, define

Assumption 1.1. Let $K$ be a set in $X_{c}^{2}$ with smooth regular boundary $M$. Assume $K$ is strongly convex if $c \geq 0$. If $c<0$, assume it is strongly $h$-convex.

Theorem 1.2. Under Assumption 1.1,

$$
\int_{M} \tan _{c}\left(\frac{\rho(s)}{2}\right) d s=F-F_{e},
$$

where $d s$ is the arclength measure on $M, F$ is the area of $K$, and $F_{e}$ is the (algebraic) area enclosed by the focal set $F(M)$ of $M$.

The convexity notions used above as well as the generalized tangent function $\tan _{c}$ will be defined next.

\section{Preliminaries}

Definition 2.1. A domain $K \subset X_{c}^{2}$ is regular if its boundary $M$ admits a regular parametrization. That is, there is an injective smooth map $\gamma: S^{1}(L) \rightarrow M$ such that $\left|\gamma^{\prime}(s)\right|=1$, where $L$ is a constant, $S^{1}(L)$ is the euclidean circle of radius $L / 2 \pi$, and $s$ is its arclength parameter.

Note that $L$ is the perimeter of $K$. By choosing a regular parametrization $\gamma$, we make $s$ the arclength parameter for $M$ as well.

Definition 2.2. A regular domain $K \subset X_{c}^{2}$ is convex if the curvature at every point of $M=\partial K$ is nonnegative; if the curvature on $M$ is always positive, $K$ is strongly convex.

The sign of the curvature can be defined using the intrinsic covariant derivative $\nabla$ of $X_{c}^{2}$ by the condition

$$
\nabla_{T} T=k N,
$$

where $N$ is the inward normal vector field and $T$ is a unit tangent vector.

Note that, if $c>0$, then $K$ lies in some half sphere of $\mathbb{S}_{c}^{2}$. If $c<0$, we need a stronger convexity notion.

Definition 2.3. A regular domain $K \subset \mathbb{H}_{c}^{2}$ with smooth boundary $M$ is said to be $h$-convex if the curvature at every point of $M$ is greater than or equal to $\sqrt{-c}$. If the same curvature is always greater than $\sqrt{-c}$, the domain is strongly $h$-convex. 
The hyperbolic disc is strongly $h$-convex because the curvature $k$ of the boundary of a disc of radius $r$ in $\mathbb{U}_{c}^{2}$ is given by

$$
k=\sqrt{-c} \operatorname{coth}(\sqrt{-c} r),
$$

and $\operatorname{coth}(t) \geq 1$ for all $t \in \mathbb{R}$.

The notion of convexity we give here is equivalent to the usual one of geodesic convexity. The $h$-convex sets are also called horocyclically convex sets, because in this case the arcs of horocycles joining points in $K$ are contained in $K$.

To deal simultaneously with the euclidean plane, the sphere, and the hyperbolic plane, we use the functions

$$
\operatorname{sn}_{c}(t):= \begin{cases}\frac{1}{\sqrt{-c}} \sinh (\sqrt{-c} t) & \text { for } c<0 \\ t & \text { for } c=0 \\ \frac{1}{\sqrt{c}} \sin (\sqrt{c} t) & \text { for } c>0\end{cases}
$$

and

$$
\mathrm{cn}_{c}(t):= \begin{cases}\cosh (\sqrt{-c} t) & \text { for } c<0 \\ 1 & \text { for } c=0 \\ \cos (\sqrt{c} t) & \text { for } c>0\end{cases}
$$

Note the identities

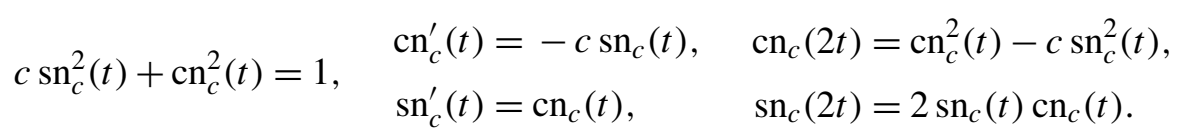

We shall use that the area and the perimeter of a disc in $X_{c}^{2}$ of radius $t$ are given respectively by

$$
A(t)=\frac{2 \pi}{c}\left(1-\mathrm{cn}_{c}(t)\right) \quad \text { and } \quad L(t)=2 \pi \operatorname{sn}_{c}(t) .
$$

Definition 2.4. Let $M$ be the boundary of a convex domain $K \subset X_{c}^{2}$ (make it $h$ convex if $c<0$ ). For each point $x \in M$ we denote by $\rho(x)$ the curvature radius of $M$ at $x$ and define it through

$$
k(x)=\cot _{c} \rho(x),
$$

where $k(x)$ is the curvature of $M$ at $x$.

Since $\operatorname{coth}(t) \geq 1$ for all $t \in \mathbb{R}$, the curvature radius when $c<0$ is only defined if $k(x) \geq \sqrt{-c}$, that is, if $K$ is $h$-convex.

Definition 2.5. Let $M$ be the boundary of a convex domain $K \subset X_{c}^{2}$ (make it $h$-convex if $c<0)$. The focal set $F(M)$ of $M$ is the set

$$
F(M)=\left\{\exp _{x}(\rho(x) N(x)) ; x \in M\right\} \subset X_{c}^{2},
$$


where $N(x)$ is the inward unit normal vector to $M$ at $x \in M$.

Recall that $y=\exp _{x}(t v)$ with $|v|=1$ means $y=\sigma(t)$ where $\sigma(s)$ is the unique geodesic such that $\sigma(0)=x$ and $\sigma^{\prime}(0)=v$.

The focal set of $M$ is also called the evolute of $M$. Note that $F(M)$ is locally smooth and that the normal geodesics to $M$ are tangent to $F(M)$.

We will see that $F(M)$ is the set of critical values of $\phi(x, t)=\exp _{x}(t N(x))$ for $x \in M$ and $t \in \mathbb{R}$.

Definition 2.6. The winding number wind $(\gamma, y)$ of a curve $\gamma: S^{1}(L) \rightarrow X_{c}^{2}$ with respect to a point $y \in X_{c}^{2} \backslash \gamma\left(S^{1}(L)\right)$ is the mapping degree of the map $\varphi: S^{1}(L) \rightarrow$ $T_{y} X_{c}^{2}$ defined by the condition $\|\varphi(s)\|=1$ and $\exp _{y} \lambda(s) \varphi(s)=\gamma(s)$ for some function $\lambda=\lambda(s)>0$.

That is, to each point $\gamma(s)$ we associate the unit tangent vector at $y$ that is tangent to the unique geodesic joining $y$ and $\gamma(s)$. We say that $\varphi$ is the winding map with respect to $y$ associated to $\gamma$. Note that $\varphi$ may be thought of as a map of $S^{1}(L)$ into $S^{1}$.

It can be seen that wind $(\gamma, y)$ is equal to the algebraic intersection number of $\gamma\left(S^{1}(L)\right)$ with an arbitrary geodesic ray emanating from $y$; see [Guillemin and Pollack 1974],

By moving $y$ along an arc that does not meet $\gamma\left(S^{1}(L)\right)$, we do not change the winding number. Hence, the winding number of $\gamma$ with respect to $y$ is constant when $y$ stays in a connected component of $X_{c}^{2} \backslash \gamma\left(S^{1}(L)\right)$. See [do Carmo 1976, p. 392].

Definition 2.7. Let $M$ be the boundary of a convex domain $K \subset X_{c}^{2}$ (make it $h$-convex if $c<0$ ) and let $y \notin M$. We define

$$
\operatorname{wind}(M, y)=\operatorname{wind}(\gamma, y),
$$

where $\gamma$ is a regular parametrization of $M$ such that the basis $\left\{\gamma^{\prime}, N\right\}$ is positive.

We define the winding number of the focal set $F(M)$ by

$$
\operatorname{wind}(F(M), y)=\operatorname{wind}(\tilde{\gamma}, y),
$$

where $\tilde{\gamma}(s)=\exp _{\gamma(s)}(\rho(s) N(s))$ is the parametrization of $F(M)$ induced by the parametrization $\gamma$ of $M$.

Once we fix the parametrization $\gamma$, we shall write $\rho(s)$ and $N(s)$ instead of $\rho(\gamma(s))$ and $N(\gamma(s))$.

The algebraic area of $F(M)$ is the area enclosed by $F(M)$, counted with sign and multiplicity. To be precise, we define the area $F_{e}$ enclosed by $F(M)$ as

$$
F_{e}=\int_{X_{c}^{2}} \operatorname{wind}(F(M), y) d y .
$$


Remark 2.8. Let $\gamma$ be a regular parametrization of the boundary $M$ of a regular domain, and let $\varphi$ be the winding map associated to $\gamma$ with respect to $y \notin M$. Let $\psi=\varphi \circ \gamma^{-1}$. Since $\operatorname{deg} \psi=\operatorname{deg} \varphi$, and because the degree theorem gives

$$
\int_{M} \psi^{*} d O_{1}=\operatorname{deg} \psi \int_{S^{1}} d O_{1}
$$

where $d O_{1}$ is the arclength measure of $S^{1}$, we have

$$
\operatorname{wind}(M, y)=\frac{1}{2 \pi} \int_{M} \psi^{*} d O_{1} .
$$

\section{An integral involving the curvature radius}

Let $M$ be the boundary of a regular domain $K \subset X_{c}^{2}$. Consider the set

$$
M_{\rho}=\cup_{x \in M}(\{x\} \times[0, \rho(x)]) \subset M \times \mathbb{R},
$$

and the map $\phi: M_{\rho} \rightarrow X_{c}^{2}$ defined by $\phi(x, t)=\exp _{x}(t N(x))$. We say that $\phi$ is the focal map of $M$. Note that $\phi$ is a (possibly) noninjective local diffeomorphism in the interior of $M_{\rho}$.

Lemma 3.1. Let $M$ be the boundary of a regular domain $K \subset X_{c}^{2}$, and let $\phi$ : $M_{\rho} \rightarrow X_{c}^{2}$ be the focal map. Then

$$
\phi^{*} d y=\left(c n_{c}(t)-k(s) s n_{c}(t)\right) d s \wedge d t,
$$

where $d y$ is the area element of $X_{c}^{2}, s$ is the arclength on $M$, and $k(s)$ is the curvature of $M$ at $\gamma(s)$.

Proof. Recall that,

$$
X_{c}^{2}= \begin{cases}S^{2}\left(\frac{1}{\sqrt{c}}\right)=\left\{(x, y, z) \in \mathbb{R}^{3}: x^{2}+y^{2}+z^{2}=\frac{1}{c}\right\} & \text { if } c>0, \\ \mathbb{M}^{2}\left(\frac{1}{\sqrt{c}}\right)=\left\{(x, y, z) \in \mathbb{R}^{(2,1)}: x^{2}+y^{2}-z^{2}=\frac{1}{c}, z>0\right\} & \text { if } c<0,\end{cases}
$$

where $\mathbb{R}^{(2,1)}$ is the Lorentz-Minkowski space.

Using these models, the focal map $\phi: M_{\rho} \rightarrow X_{c}^{2}$ is given in coordinates by

$$
\phi(s, t)=\mathrm{cn}_{c}(t) \gamma(s)+\operatorname{sn}_{c}(t) N(s) \quad \text { for all } c \in \mathbb{R},
$$

where $\gamma: S^{1}(L) \rightarrow X_{c}^{2}$ is a regular parametrization of $M$; see [Ratcliffe 1994].

On the other hand, since $d y$ is a 2 -form in $X_{c}^{2}$, there is a function $p=p(s, t)$ such that $\phi^{*} d y=p(s, t) d s \wedge d t$.

Let us compute $p(s, t)$. Recall

$$
\nabla_{\gamma^{\prime}} \gamma^{\prime}=k N \quad \text { and } \quad \nabla_{\gamma^{\prime}} N=-k \gamma^{\prime},
$$


where $\nabla$ is the intrinsic covariant derivative of $X_{c}^{2}$ and $N$ is the inward normal vector field.

We have

$$
\begin{aligned}
p(s, t) & =\phi^{*} d y\left(\frac{\partial}{\partial s}, \frac{\partial}{\partial t}\right)=d y\left(\phi_{*}\left(\frac{\partial}{\partial s}\right) \phi_{*}\left(\frac{\partial}{\partial t}\right)\right) \\
& =d y\left(\left(\operatorname{cn}_{c}(t)-k(s) \operatorname{sn}_{c}(t)\right) \gamma^{\prime}(s),-c \operatorname{sn}_{c}(t) \gamma(s)+\operatorname{cn}_{c}(t) N(s)\right) .
\end{aligned}
$$

Let $\eta$ be the volume element of $\mathbb{R}^{3}$ if $c>0$ or of $\mathbb{R}^{2,1}$ if $c<0$. Then $d y$ is the contraction of $\eta$ with the normal vector field to $S^{2}(1 / \sqrt{c})$ or $\mathbb{M}^{2}(1 / \sqrt{c})$, respectively. In both cases, the outward normal to $X_{c}^{2}$ at the point $\phi(s, t)$ is the vector $\phi(s, t)$. Hence $d y_{\phi(s, t)}=\sqrt{c} i_{\phi(s, t)} \eta$. Note that $\eta\left(\gamma, \gamma^{\prime}, N\right)=1 / \sqrt{c}$. Hence

$$
\begin{aligned}
p(s, t)= & \sqrt{c} \eta\left(\mathrm{cn}_{c}(t) \gamma(s)+\operatorname{sn}_{c}(t) N(s),\left(\operatorname{cn}_{c}(t)-k(s) \operatorname{sn}_{c}(t)\right) \gamma^{\prime}(s),\right. \\
& \left.-c \operatorname{sn}_{c}(t) \gamma(s)+\operatorname{cn}_{c}(t) N(s)\right) \\
= & \sqrt{c} \eta\left(\operatorname{cn}_{c}(t) \gamma(s),\left(\operatorname{cn}_{c}(t)-k(s) \operatorname{sn}_{c}(t)\right) \gamma^{\prime}(s), \operatorname{cn}_{c}(t) N(s)\right)+ \\
& \quad \sqrt{c} \eta\left(\operatorname{sn}_{c}(t) N(s),\left(\operatorname{cn}_{c}(t)-k(s) \operatorname{sn}_{c}(t)\right) \gamma^{\prime}(s),-c \operatorname{sn}_{c}(t) \gamma(s)\right) \\
= & \sqrt{c}\left(\operatorname{cn}_{c}(t)-k(s) \operatorname{sn}_{c}(t)\right)\left(\operatorname{cn}_{c}^{2}(t)+c \operatorname{sn}_{c}^{2}(t)\right) \eta\left(\gamma(s), \gamma^{\prime}(s), N(s)\right) \\
= & \left(\operatorname{cn}_{c}(t)-k(s) \operatorname{sn}_{c}(t)\right) .
\end{aligned}
$$

Finally, if $c=0$, we have $p(s, t)=d y\left((1-t k(s)) \gamma^{\prime}, N\right)=(1-t k(s))$.

Remark 3.2. Observe that $p(s, t)=c n_{c}(t)-k(s) s n_{c}(t) \geq 0$ if and only if $\cot _{c} \rho(s)=k(s) \leq \cot _{c}(t)$, that is, if and only if $t \leq \rho(s)$. This is the situation in the hypothesis of Lemma 3.1.

Definition 3.3. Let $K$ be a convex set in $X_{c}^{2}$ with smooth regular boundary $M$, and let $y \in X_{c}^{2}$. Let $h_{y}: M \rightarrow \mathbb{R}$ be the distance function to $y$, that is, $h_{y}(x)=d(x, y)$. Let $x \in M$ be a critical point of $h_{y}$. We say that $x$ is a $\rho$-critical point of $h_{y}$ if $d(x, y) \leq \rho(x)$, where $\rho(x)$ is the curvature radius of $M$ at $x$.

Note that if $x$ is a $\rho$-critical point of $h_{y}$, then $y=\exp _{x}(t N(x))$ with $0 \leq t \leq \rho(x)$.

Theorem 3.4. Under Assumption 1.1,

$$
\int_{X_{c}^{2}} v_{\rho}(y) d y=\int_{M} \tan _{c}\left(\frac{\rho(s)}{2}\right) d s,
$$

where $v_{\rho}(y)$ is the number of $\rho$-critical points of the distance function $h_{y}, s$ is the arclength of $M$, and $\rho(s)$ is the curvature radius of $M$ at $\gamma(s)$.

Proof. Applying the coarea formula to the focal map $\phi$, we have

$$
\int_{\phi\left(M_{\rho}\right)} \#\left(\phi^{-1}(y)\right) d y=\int_{M_{\rho}}\left|\phi^{*} d y\right| .
$$


Because of its construction, $\phi$ catches each point $y \in \phi\left(M_{\rho}\right)$ exactly $v_{\rho}(y)$ times. Moreover, since $\#\left(\phi^{-1}(y)\right)=0$ for $y \notin \phi\left(M_{\rho}\right)$, we have

$$
\int_{X_{c}^{2}} v_{\rho}(y) d y=\int_{M_{\rho}}\left|\phi^{*} d y\right| .
$$

By Remark 3.2 we have $\left|\phi^{*} d y\right|=\phi^{*} d y$, and hence

$$
\begin{aligned}
\int_{X_{c}^{2}} v_{\rho}(y) d y & =\int_{M_{\rho}} \phi^{*} d y=\int_{M} \int_{0}^{\rho(s)} p(s, t) d t d s \\
& =\int_{M} \int_{0}^{\rho(s)}\left(\operatorname{cn}_{c}(t)-k(s) \operatorname{sn}_{c}(t)\right) d t d s \\
& =-\frac{1}{c} \int_{M}\left(-c \operatorname{sn}_{c}(\rho(s))+k(s)\left(1-\operatorname{cn}_{c}(\rho(s))\right)\right) d s \\
& =-\frac{1}{c} \int_{M}\left(-c \operatorname{sn}_{c}(\rho(s))+\cot _{c}(\rho(s))\left(1-\operatorname{cn}_{c}(\rho(s))\right)\right) d s \\
& =-\frac{1}{c} \int_{M} \frac{\operatorname{cn}_{c}(\rho(s))-1}{\operatorname{sn}_{c}(\rho(s))} d s=\int_{M} \tan _{c} \frac{\rho(s)}{2} d s .
\end{aligned}
$$

Remark 3.5. Note that $A(\rho(s)) / L(\rho(s))=\tan _{c}(\rho(s) / 2)$, where $A(\rho(s))$ and $L(\rho(s))$ are respectively the area and the length of the disc of radius $\rho(s)$ in $X_{c}^{2}$.

Thus, we have proved

$$
\int_{X_{c}^{2}} v_{\rho}(y) d y=\int_{M} \frac{A(\rho(s))}{L(\rho(s))} d s .
$$

Lemma 3.6. Adopt Assumption 1.1. Let $y \in K$, and let $x \in M$ be a minimum of the function $h_{y}$. Then $x$ is a $\rho$-critical point of $h_{y}$.

Proof. Let $\gamma(s)$ be an arclength parametrization of $M$. Consider $f(s)=h_{y}(\gamma(s))$. If $s_{0}$ is such that $\gamma\left(s_{0}\right)=x$, we have $f^{\prime}\left(s_{0}\right)=0$ and $f^{\prime \prime}\left(s_{0}\right)>0$. Now $f^{\prime}(s)=$ $g\left(X, \gamma^{\prime}(s)\right)$, where $g$ is the metric on $X_{c}^{2}$, and $X=\operatorname{grad}(d(\cdot, y))$ is the gradient field (over $X_{c}^{2}$ ) of the distance function to $y$. Then

$$
\begin{aligned}
0<f^{\prime \prime}\left(s_{0}\right) & =\gamma^{\prime}\left(g\left(X, \gamma^{\prime}(s)\right)\left(s_{0}\right)\right. \\
& =g\left(\nabla_{\gamma^{\prime}} X, \gamma^{\prime}(s)\right)\left(s_{0}\right)+g\left(X, \nabla_{\gamma^{\prime}} \gamma^{\prime}\right)\left(s_{0}\right)=k_{f}-k\left(s_{0}\right),
\end{aligned}
$$

where $k_{f}=\cot _{c}\left(f\left(s_{0}\right)\right)$ is the geodesic curvature of the circle through $x$ with center $y$. Thus, we have $\cot _{c}(\rho(x))<\cot _{c}\left(h_{y}(x)\right)$, which implies $\rho(x)>d(x, y)$; thus $x$ is a $\rho$-critical point.

Lemma 3.7. Under Assumption 1.1, we have

$$
v_{\rho}(y)=\operatorname{wind}(M, y)-\operatorname{wind}(F(M), y) \quad \text { for } y \notin M \cup F(M) .
$$

Proof. Let $\phi: M_{\rho} \rightarrow X_{c}^{2}$ be the focal map of $M$, that is, $\phi(x, t)=\exp _{x}(t N(x))$. 
Following [White 1970], we put

$$
I=\left\{n \in M_{\rho} ; y=\phi(n)\right\}=\phi^{-1}(y),
$$

for a fixed generic point $y \in X_{c}^{2} \backslash(M \cup F(M))$; the last ensures $I$ is finite. We define

$$
e: M_{\rho}-I \rightarrow T_{y}^{1} X_{c}^{2}
$$

by the condition $\|e(n)\|=1$ and by $\exp _{y} \lambda(n) e(n)=\phi(n)$, for some function $\lambda(n)$. Let $I_{\epsilon}=\bigcup_{i \in I} C_{i}$, where $C_{i}$ are small, disjoint discs surrounding the points $i \in I$.

Applying Stokes' theorem to the punctured manifold $M_{\rho}-I_{\epsilon}$, we obtain

$$
0=\int_{M_{\rho}-I_{\epsilon}} e^{*} d\left(d O_{1}\right)=\int_{\partial\left(M_{\rho}-I_{\epsilon}\right)} e^{*} d O_{1} .
$$

Note that $\partial\left(M_{\rho}-I_{\epsilon}\right)=M \cup M_{e} \cup \bigcup_{i} \partial\left(C_{i}\right)$, where $M_{e}=\{(x, \rho(x)) ; x \in M\}$. Note also that $\phi\left(M_{e}\right)=F(M)$.

Because $e$ is an orientation-preserving local diffeomorphism, all the integrals $\int_{\partial\left(C_{i}\right)} e^{*} d O_{1}$ are equal to $2 \pi$. Hence, taking into account the orientations induced at the boundary, we have

$$
\left.\int_{M} e^{*}\right|_{M} d O_{1}-\left.\int_{M_{e}} e^{*}\right|_{M_{e}} d O_{1}-2 \pi \#(I)=0
$$

But $\# I=v_{\rho}(y)$, so

$$
\left.\frac{1}{2 \pi} \int_{M} e^{*}\right|_{M} d O_{1}-\left.\frac{1}{2 \pi} \int_{M_{e}} e^{*}\right|_{M_{e}} d O_{1}=v_{\rho}(y) .
$$

Now we fix a regular parametrization $\gamma: S^{1}(L) \rightarrow M$. It is clear that $\left.e^{*}\right|_{M} \circ \gamma$ is the winding map with respect to $y$ associated to $\gamma$. It follows from Remark 2.8 that

$$
\operatorname{wind}(M, y)=\left.\frac{1}{2 \pi} \int_{M} e^{*}\right|_{M} d O_{1} .
$$

Analogously, let $j: S^{1}(L) \rightarrow M_{\rho}$ be the map $j(s)=(s, \rho(s))$, and let $\tilde{\gamma}$ be the parametrization of $F(M)$ induced by the parametrization of $M$. Then $\tilde{\varphi}=\left.e^{*}\right|_{M_{e}} \circ j$ is the winding map with respect to $y$ associated to $\tilde{\gamma}$. Note that $j\left(S^{1}(L)\right)=M_{e}$. Thus

$$
\operatorname{wind}(F(M), y)=\operatorname{wind}(\tilde{\gamma}, y)=\operatorname{deg} \tilde{\varphi}=\left.\operatorname{deg} e^{*}\right|_{M_{e}}=\left.\frac{1}{2 \pi} \int_{M_{e}} e^{*}\right|_{M_{e}} d O_{1} .
$$

Hence, for each $y \in X_{c}^{2} \backslash(M \cup F(M))$, equality (4) becomes

$$
\operatorname{wind}(M, y)-\operatorname{wind}(F(M), y)=v_{\rho}(y) .
$$


Theorem 3.8. Under Assumption 1.1, we have

$$
\int_{M} \tan _{c}\left(\frac{\rho(s)}{2}\right) d s=F-F_{e},
$$

where $s$ is the arclength of $M, F$ is the area of $K$, and $F_{e}$ is the (algebraic) area of the focal set $F(M)$ of $M$.

Proof. From Theorem 3.4 and Lemma 3.7 we have

$$
\int_{M} \tan _{c}\left(\frac{\rho(s)}{2}\right) d s=\int_{X_{c}^{2}}(\operatorname{wind}(M, y)-\operatorname{wind}(F(M), y)) d y .
$$

$\operatorname{But} \operatorname{wind}(M, y)=1$ if $y \in K$, and $\operatorname{wind}(M, y)=0$ if $y \notin K$. The (algebraic) area of $F(M)$ is, by definition, the integral over $X_{c}^{2}$ of the winding number of $F(M)$ with respect to every $y \in X_{c}^{2}$.

We also obtain a generalization of formula (1).

Corollary 3.9. Under Assumption 1.1, we have

$$
\int_{M} \tan _{c}\left(\frac{\rho(s)}{2}\right) d s \geq F
$$

Equality holds if and only if $M$ is a circle.

Proof. The inequality (5) is a consequence of $v_{\rho}(y) \geq \operatorname{wind}(M, y)$, which is evident because $y \notin K$ implies $\operatorname{wind}(M, y)=0$, whereas $y \in K$ implies $v_{\rho}(y) \geq 1$. Note that this proves wind $(F(M), y) \leq 0$ and $F_{e} \leq 0$. If $M$ is a circle and $F(M)$ is its center, then $F_{e}=0$, and we have equality in (5).

Finally, if equality holds in (5), we have

$$
\int_{Y} \operatorname{wind}(F(M), y) d y=0 .
$$

Since wind $(F(M), y) \leq 0$, it must be that wind $(F(M), y)=0$ almost everywhere.

If $F(M)$ were not a point we could choose a small ball separated by $F(M)$ in two connected components. The winding number is a different integer in each of these parts, which gives a contradiction.

Remark 3.10. If $c=0$ we have $\int_{M} \rho(s) d s \geq 2 F$.

This, together with Theorem 3.8 for $c=0$, gives $F_{e} \leq 0$, which is also a consequence of the Wirtinger inequality. Indeed, that inequality states that if $f: \mathbb{R} \rightarrow \mathbb{R}$ is a $C^{2}$-function of period $2 \pi$, then

$$
\int_{0}^{2 \pi}\left|f^{\prime}\right|^{2} d \phi \leq \int_{0}^{2 \pi}\left|f^{\prime \prime}\right|^{2} d \phi
$$

Equality holds if and only if $f(\phi)=a \cos \phi+b \sin \phi+c$ for constants $a, b$, and $c$. See, for instance, [Hopf 1983, p. 52]. 
It was seen in [Escudero and Reventós 2007] that

$$
F_{e}=\frac{1}{2} \int_{0}^{2 \pi}\left(p^{\prime 2}-p^{\prime \prime 2}\right) d \phi
$$

where $p(\phi)$ is the support function of the convex set; hence (6) implies $F_{e} \leq 0$.

Conversely, $F_{e} \leq 0$ for an arbitrary convex set implies (6). Indeed, given $f$ we consider $p=f+c$ with $c$ constant so that $p+p^{\prime \prime}>0$. Now we apply $F_{e} \leq 0$ to the convex set with support function $p$.

Thus we have a geometrical interpretation of the Wirtinger inequality: every convex set $K$ is covered by the geodesic segments joining each point of $\partial K$ to the corresponding curvature center.

\section{The integral of $\tan _{c} \rho(s)$}

Note that, in the case $c=0$, Theorem 3.8 gives $\int_{M} \rho(s) / 2 d s=F-F_{e}$, which is formula (2). It can also be written as

$$
\frac{1}{2} \int_{M} \frac{1}{k(s)} d s=F-F_{e},
$$

and, since in $X_{c}^{2}$ the relation between the curvature $k(s)$ and the curvature radius $\rho(s)$ is given by $k(s)=\cot _{c} \rho(s)$, it seems interesting to estimate

$$
\int_{M} \tan _{c} \rho(s) d s
$$

For this, we recall the Gauss-Bonnet theorem [Santaló 1976, p. 303]

$$
\int_{M} k(s) d s+c F=2 \pi
$$

and the isoperimetric inequality [p. 324]

$$
L^{2}+c F^{2}-4 \pi F \geq 0 .
$$

We apply these to the convex set $K(M=\partial K)$ of area $F$ and perimeter $L$ in $X_{c}^{2}$ :

$$
\begin{aligned}
4 \pi F-c F^{2} \leq L^{2} & =\left(\int_{M} \sqrt{\cot _{c}(\rho(s))} \sqrt{\tan _{c}(\rho(s))} d s\right)^{2} \\
& \leq \int_{M} \cot _{c}(\rho(s)) d s \int_{M} \tan _{c}(\rho(s)) d s \\
& =\int_{M} k(s) d s \int_{M} \tan _{c}(\rho(s)) d s=(2 \pi-c F) \int_{M} \tan _{c}(\rho(s)) d s .
\end{aligned}
$$

Hence we have 
Theorem 4.1. Under Assumption 1.1,

$$
\int_{M} \tan _{c} \rho(s) d s \geq F \frac{4 \pi-c F}{2 \pi-c F}
$$

Equality holds if and only if $M$ is a circle.

\section{Remark 4.2. Since}

$$
1<\frac{4 \pi-c F}{2 \pi-c F} \leq 2
$$

we have

$$
\int_{M} \tan _{c} \rho(s) d s>F .
$$

This also follows directly from formula (5).

\section{Acknowledgments}

We would like to thank professors E. Gallego and E. Teufel for many helpful conversations during the preparation of this work.

\section{References}

[do Carmo 1976] M. P. do Carmo, Differential geometry of curves and surfaces, Prentice-Hall, Englewood Cliffs, N.J., 1976. Translated from the Portuguese. MR 52 \#15253 Zbl 1028.34017

[Escudero and Reventós 2007] C. A. Escudero and A. Reventós, "An interesting property of the evolute", Amer. Math. Monthly 114:7 (2007), 623-628. MR 2341325

[Escudero and Rodríguez 1996] C. A. Escudero and C. Rodríguez, Curvatura en un poligono y teorema de Ros para curvas planas, Master's thesis, Universidad del Valle, Colombia, 1996.

[Gallego et al. 2005] E. Gallego, A. Reventós, G. Solanes, and E. Teufel, "Width of convex bodies in spaces of constant curvature", Preprint 34, Departament de Matemàtiques, Univ. Autònoma de Barcelona, 2005.

[Guillemin and Pollack 1974] V. Guillemin and A. Pollack, Differential topology, Prentice-Hall, Englewood Cliffs, N.J., 1974. MR 50 \#1276 Zbl 0361.57001

[Hopf 1983] H. Hopf, Differential geometry in the large, Lecture Notes in Mathematics 1000, Springer, Berlin, 1983. MR 85b:53001 Zbl 0526.53002

[Osserman 1990] R. Osserman, "Curvature in the eighties", Amer. Math. Monthly 97:8 (1990), 731756. MR 91i:53001 Zbl 0722.53001

[Ratcliffe 1994] J. G. Ratcliffe, Foundations of hyperbolic manifolds, Graduate Texts in Mathematics 149, Springer, New York, 1994. MR 95j:57011 Zbl 0809.51001

[Ros 1988] A. Ros, "Compact hypersurfaces with constant scalar curvature and a congruence theorem”, J. Differential Geom. 27:2 (1988), 215-223. MR 89b:53096 Zbl 0638.53051

[Santaló 1976] L. A. Santaló, Integral geometry and geometric probability, Addison-Wesley, Reading, MA-London-Amsterdam, 1976. MR 55 \#6340 Zbl 0342.53049

[White 1970] J. H. White, "Some differential invariants of submanifolds of Euclidean space", $J$. Differential Geometry 4 (1970), 207-223. MR 42 \#1035 Zbl 0198.53501

[Zhou 2007] J. Zhou, "Curvature inequalities for curves", 2007. To appear in Int. J. Math. Sci. (India). 
Received December 15, 2006. Revised June 26, 2007.

CARlos ARturo Escudero

Departamento de Matemáticas

Universidad Tecnológica de Pereira

A.A.097 PEREIRA, RisARALDA

COLOMBIA

carlos10@utp.edu.co

Agustí ReVEntós

DEPARTAMENT DE MATEMÀtiques

UNIVERSITAT AUTÒNOMA DE BARCELONA

08193 BELLATERRA (CERDANYOLA DEL VALLÈS)

SPAIN

agusti@mat.uab.es

Gil SOLANES

DePartament D’ Àlgebra i GeOMETRIA

UNIVERSITAT DE BARCELONA

GRAN Via DE LES CORTS CATALANES 585

08007 BARCELONA

SPAIN

solanes@ub.edu 